\title{
Hadron-Induced Radiation Damage in LuAG:Ce Scintillating Ceramics
}

\author{
Chen Hu, Member, IEEE, Liyuan Zhang, Member, IEEE, Ren-Yuan Zhu, Senior Member, IEEE, Jiang Li, \\ Benxue Jiang, Jon Kapustinsky, Senior Member, IEEE, Michael Mocko, Ron Nelson, Xuan Li, Zhehui Wang
}

\begin{abstract}
Because of its potential low cost, bright light and fast decay time LuAG:Ce ceramic scintillators have attracted a broad interest in the high energy physics community. One crucial issue for its application in future high energy physics experiments is its radiation hardness against neutrons and protons expected at future hadron colliders. We report optical and scintillation performance of $1 \mathrm{~mm}$ LuAG:Ce ceramic samples doped with $\mathrm{Mg}^{2+}$ $\left(\right.$ and $\mathrm{Ca}^{2+}$ ) and their radiation damage induced by hadrons. While $\mathrm{Mg}^{2+}$ co-doping improves their light output, $\mathrm{Ca}^{2+}$ co-doping improves their F/T ratio. LuAG:Ce ceramic samples were irradiated at LANSCE by neutrons up to $6.7 \times 10^{15} \mathrm{neq}_{\mathrm{eq}} / \mathrm{cm}^{2}$, and by $24 \mathrm{GeV}$ and $800 \mathrm{MeV}$ protons at CERN PS-IRRAD up to $1.2 \times 10^{15}$ $\mathrm{p} / \mathrm{cm}^{2}$ and at LANSCE up to $2.3 \times 10^{14} \mathrm{p} / \mathrm{cm}^{2}$, respectively. All samples show excellent radiation hardness with more than $90 \%$ of light after irradiation. The RIAC values induced by neutrons are found to be a factor of two smaller than LYSO:Ce crystals. The RIAC values induced by protons are also found a factor of two smaller than LYSO:Ce crystals in LuAG:Ce ceramic samples with good optical quality. R\&D will continue to develop LuAG:Ce scintillating ceramics with improve optical quality for future investigation.
\end{abstract}

\section{INTRODUCTION}

$\mathrm{B}_{\mathrm{s}}$ RIGHT and fast cerium doped $\mathrm{Lu}_{3} \mathrm{Al}_{5} \mathrm{O}_{12}$ :Ce ceramic scintillators (LuAG:Ce) have attracted a broad interest in the high energy physics (HEP) community. Thanks to the development of nanotechnology, by using high purity nanoparticles as starting powders [1-3], polycrystalline cubic LuAG ceramics without intrinsic birefringence may be fabricated as fine-grained, pore-free, and thus transparent scintillator with optical quality comparable to their single crystal counterparts $[4,5]$. Our previous work revealed that LuAG:Ce ceramic samples show a high light output of 1,400 photoelectron/MeV (p.e./MeV) measured by a Hamamatsu R2056 PMT with bialkali cathode, a fast primary decay time of $50 \mathrm{~ns}$, and are radiation hard against an ionization dose up to 2.2 MGy and a proton fluence up to $3 \times 10^{14} \mathrm{p} / \mathrm{cm}^{2}$ [6]. A slow scintillation component with a decay time of about $1 \mu$ s may also be suppressed by alkaline earth metal co-doping, such as $\mathrm{Mg}^{2+}[7,8]$ and $\mathrm{Ca}^{2+}[6,9]$ co-doping. The fast to total ratio $(\mathrm{F} / \mathrm{T})$, defined as the ratio between light output in 200 and 3,000 ns gate, may reach $90 \%$ with $\mathrm{Ca}^{2+}$ co-doping [6].

Manuscript received Dec 12, 2021. This work was supported in part by the US Department of Energy Grants DE-SC0011925 and DE-AC52-06NA25396. C. Hu, L. Zhang, and R.-Y. Zhu are with the HEP, California Institute of Technology, Pasadena, CA 91125, USA (e-mail: zhu@hep.caltech.edu).

$\mathrm{J}$. Li is with the Shanghai Institute of Ceramics, Chinese Academy of Sciences, Shanghai, 200050, China
Because of a simpler production technology at a lower temperature, a higher raw material usage and no need for after growth mechanical processing, LuAG:Ce ceramic scintillators may be more cost-effective in mass production as compared to widely used cerium doped lutetium-yttrium oxyorthosilicate (LYSO:Ce) crystals. This material therefore is promising for future HEP experiments in a severe radiation environment, such as the High-Luminosity Large Hadron Collider (HL-LHC) and the Future Hadron Circular Collider (FCC-hh). One possible application would be to replace LYSO:Ce crystal plates as the active material for a shashlik sampling calorimeter for future HEP experiments [6].

One crucial issue, however, is its radiation hardness against hadrons. With a $5 \times 10^{34} \mathrm{~cm}^{-2} \mathrm{~s}^{-1}$ luminosity and a $3,000 \mathrm{fb}^{-1}$ integrated luminosity, the HL-LHC will present a radiation environment, where up to $1 \mathrm{MGy}$ ionization dose, $6 \times 10^{14}$ charged hadrons $/ \mathrm{cm}^{2}$ and $3 \times 10^{15}$ fast neutron $/ \mathrm{cm}^{2}$ are expected for the endcap calorimeter [10]. Although radiation hardness against ionization dose $[6,9,11-13]$ and protons $[6,13]$ was reported for LuAG:Ce crystals and ceramics, investigations are ongoing to understand radiation damage induced by hadrons, including both neutrons and protons, in LuAG:Ce ceramic samples.

$\mathrm{Mg}^{2+}$ (and $\mathrm{Ca}^{2+}$ ) co-doped LuAG:Ce ceramic samples were irradiated by neutrons at the East Port of the Weapons Neutron Research facility of Los Alamos Neutron Science Center (LANSCE) up to a $1 \mathrm{MeV}$ equivalent neutron fluence of $6.7 \times 10^{15} \mathrm{n}_{\mathrm{eq}} / \mathrm{cm}^{2}$. LuAG:Ce ceramic samples were also irradiated up to $1.2 \times 10^{15} \mathrm{p} / \mathrm{cm}^{2}$ by $24 \mathrm{GeV}$ protons at the CERN PS-IRRAD and up to $2.3 \times 10^{14} \mathrm{p} / \mathrm{cm}^{2}$ by $800 \mathrm{MeV}$ protons at the Blue Room of LANSCE, respectively. Their optical and scintillation properties were measured before and after irradiation at the Caltech HEP Crystal Laboratory with corresponding degradations compared to LYSO:Ce crystals. The effect of $\mathrm{Mg}^{2+}$ and $\mathrm{Ca}^{2+}$ co-doping on slow component suppression and radiation hardness is also discussed.

B. Jiang is with the Shanghai Institute of Optics and Fine Mechanics, Chinese Academy of Sciences, Shanghai, 201800, China

J. Kapustinsky, M. Mocko, R. Nelson, X. Li, and Z. Wang are with the Physics Department, Los Alamos National Laboratory, Los Alamos, NM 87545, USA 
TABLE I

LUAG:CE CERAMIC SCINTILLATOR SAMPLES USED IN THE NEUTRON AND PROTON IRRADIATION EXPERIMENTS

\begin{tabular}{cccccccc}
\hline \hline Sample ID & $\begin{array}{c}\text { Dimension } \\
\left(\mathrm{mm}^{3}\right)\end{array}$ & $\begin{array}{c}\text { EWLT } \\
(\%)\end{array}$ & $\begin{array}{c}200 \mathrm{~ns} \text { L.O. } \\
(\text { p.e./MeV) }\end{array}$ & $\begin{array}{c}\text { F/T ratio } \\
(\%)\end{array}$ & Experiment & $\begin{array}{c}\text { Fluence } \\
\left(\mathrm{cm}^{-2}\right)\end{array}$ & $\begin{array}{c}\text { Cooling Time } \\
(\text { days })\end{array}$ \\
\hline $\mathrm{n}-1$ & $\Phi 14.4 \times 1$ & 52.7 & 1474 & 66.6 & LANSCE-7638 & $1.7 \times 10^{15}$ & 180 \\
$\mathrm{n}-2$ & $\Phi 14.4 \times 1$ & 61.5 & 1479 & 65.6 & LANSCE-7638 & $3.4 \times 10^{15}$ & 145 \\
$\mathrm{n}-3$ & $\Phi 14.4 \times 1$ & 62.8 & 1514 & 73.5 & LANSCE-7638 & $6.7 \times 10^{15}$ & 85 \\
$\mathrm{p}-1$ & $\Phi 14.4 \times 1$ & 67.6 & 1486 & 74.2 & CERN PS-IRRAD & $7.1 \times 10^{13}$ & 339 \\
$\mathrm{p}-2$ & $\Phi 14.4 \times 1$ & 14.2 & 1305 & 62.5 & CERN PS-IRRAD & $3.6 \times 10^{14}$ & 341 \\
$\mathrm{p}-3$ & $\Phi 14.4 \times 1$ & 32.1 & 1283 & 61.6 & CERN PS-IRRAD & $1.2 \times 10^{15}$ & 351 \\
$\mathrm{p}-4$ & $\Phi 17 \times 1$ & 9.3 & 1013 & 88.0 & LANSCE-8051 & $2.4 \times 10^{13}$ & 260 \\
$\mathrm{p}-5$ & $\Phi 17 \times 1$ & 20.3 & 1049 & 89.0 & LANSCE-8051 & $2.3 \times 10^{14}$ & 259 \\
\hline \hline
\end{tabular}

\section{EXPERIMENTAL DETAILS}

The top row of Fig. 1 shows three LuAG:Ce,Mg samples of $\Phi 14.4 \times 1 \mathrm{~mm}^{3}, \mathrm{n}-1, \mathrm{n}-2$, and $\mathrm{n}-3$, from Shanghai Institute of Ceramics (SIC) for the neutron irradiation. The bottom row of Fig. 1 shows three LuAG:Ce,Mg ceramic samples of $\Phi 14.4 \times 1$ $\mathrm{mm}^{3}$, p-1, p-2, and p-3, from SIC and two LuAG:Ce,Ca, Mg samples of $\Phi 17 \times 1 \mathrm{~mm}^{3}$, p-4 and p-5, from Shanghai Institute of Optics and Fine Mechanics (SIOM) used in the proton irradiation. All samples were prepared by a solid-state reaction method. They were sintered above $1,800{ }^{\circ} \mathrm{C}$ in vacuum, followed by annealing to eliminate point defects, such as the oxygen vacancies. The details for the fabrication process can be found elsewhere [14, 15]. As shown in the transmittance spectra below, all samples show no obvious change of appearance after irradiation.

All available samples were used in this investigation without preselection. Development of transparent LuAG:Ce ceramics with excellent optical quality, however, requires extensive R\&D. One crucial issue is optical quality. Porosity as low as $0.09 \%$ may results in a more than $30 \%$ drop in transmittance for $1 \mathrm{~mm}$ ceramic samples [16].

\section{Neutron Irradiation Samples}

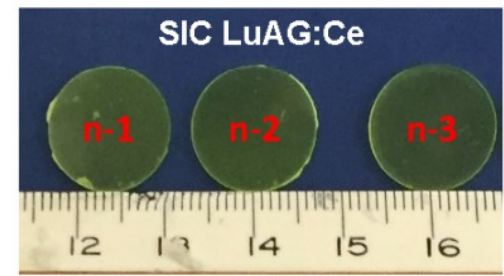

\section{Proton Irradiation Samples}
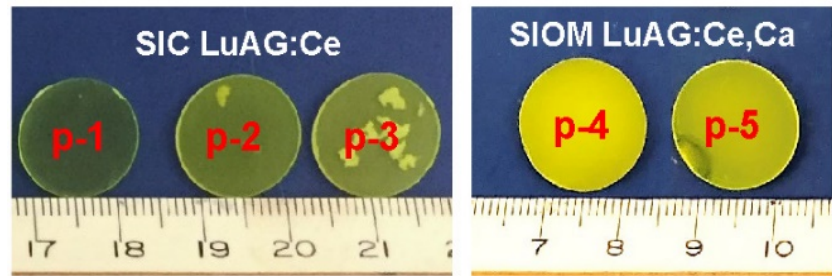

Fig. 1. Photos of three LuAG:Ce,Mg samples of $\Phi 14.4 \times 1 \mathrm{~mm}^{3}$ from SIC used for the neutron irradiation (top), three LuAG:Ce,Mg samples of $\Phi 14.4 \times 1 \mathrm{~mm}^{3}$ from SIC and two LuAG:Ce,Ca,Mg samples of $\Phi 17 \times 1 \mathrm{~mm}^{3}$ from SIOM for the proton irradiation (bottom).
We notice that samples p2-p5 show poorer transparency as compared to other samples used in this investigation, which would lead to a larger uncertainty in the radiation induced absorption coefficient data.

Three SIC LuAG:Ce,Mg samples were irradiated by neutrons in the Target 4 area in the neutron irradiation experiment LANSCE-7638. They were inside a sample holder at about $1.2 \mathrm{~m}$ away from the neutron production target in the East Port of LANSCE. The detailed description for the neutron irradiation site can be found elsewhere [17]. In addition, three groups of LYSO and $\mathrm{BaF}_{2}$ crystals prepared by SIC were also irradiated at the same time together with the ceramic samples to facilitate a direct comparison. Two samples were used in each group for $\mathrm{LYSO}$ and $\mathrm{BaF}_{2}$ crystals, so the corresponding results shown in this paper are the average of two samples.

Three SIC LuAG:Ce,Mg samples were irradiated at the CERN PS-IRRAD Proton Facility by $24 \mathrm{GeV}$ protons with a Gaussian shape and a full width at half maximum (FWHM) of about $12 \mathrm{~mm}$. Two SIOM LuAG:Ce,Ca,Mg samples were irradiated in the Blue Room of LANSCE in the proton irradiation experiment LANSCE-8051 by $800 \mathrm{MeV}$ protons with a FWHM of about $25 \mathrm{~mm}$.

Table I summarizes the optical and scintillation performance for these samples before irradiation, the hadron fluence and the cooling time after irradiation. Compared to LuAG:Ce ceramic samples without co-doping, the $\mathrm{Mg}^{2+}$ co-doped LuAG:Ce samples show a higher light output, and the $\mathrm{Ca}^{2+}$ and $\mathrm{Mg}^{2+}$ codoped samples show a higher F/T ratio. LuAG:Ce samples after hadron irradiation were radioactive. A cooling down time thus is required before they are safe to be shipped back to Caltech.

The neutron flux as a function of the neutron energy was calculated by using a Monte Carlo N-Particle eXtended (MCNPX) transport simulation package [18] developed by LANSCE. The corresponding $1 \mathrm{MeV}$ equivalent neutron fluence was calculated according to the nonionizing energy loss (NIEL) cross section as a function of the neutron energy [19]. The details of these calculations and the neutron irradiation experiment can be found in our previous paper on this subject [17]. The uncertainty of the neutron fluence is about $10 \%$. The proton fluence at CERN PS-IRRAD was measured by 
dosimeters of $10 \times 10$ and $20 \times 20 \mathrm{~mm}^{3}$. The errors of the proton fluence at CERN and LANSCE are $7 \%$ and $10 \%$, respectively.

All samples were wrapped with aluminum foil to avoid optical bleaching. They were stored at room temperature after irradiation and were measured after cooling down. Since radiation damage in LuAG:Ce ceramic samples does not recover at room temperature, the measured damage level is not affected by the cooling time.

The transmittance was measured by using a Hitachi U3210 spectrophotometer with $0.2 \%$ precision. The emissionweighted longitudinal transmittance (EWLT) was calculated according to

$$
E W L T=\frac{\int T(\lambda) E m(\lambda) d \lambda}{\int E m(\lambda) d \lambda}
$$

where $T(\lambda)$ and $\operatorname{Em}(\lambda)$ are transmittance and emission spectra. The EWLT value provides a numerical representation of the transmittance over the entire emission spectrum. The RIAC value was calculated as

$$
R I A C=\frac{1}{l} \ln \left(\frac{T_{0}}{T_{1}}\right)
$$

where $l$ is the light path length along which the transmittance was measured. $T_{0}$ and $T_{1}$ are the transmittance values at $510 \mathrm{~nm}$, which is the LuAG:Ce emission peak, measured before and after irradiation respectively. The precision of the RIAC value depends on the light path length and its initial transmittance. It is about $5 \mathrm{~m}^{-1}$ for $1 \mathrm{~mm}$ thick samples with good initial transparency, such as samples $\mathrm{n}-1, \mathrm{n}-2, \mathrm{n}-3$, and $\mathrm{p}-1$. For samples with poor initial transparency, such as p-2, p-3, p-4 and $\mathrm{p}-5$, the precision of the RIAC data would be larger than $5 \mathrm{~m}^{-1}$.

The light output before and after irradiation was measured by a Hamamatsu R2059 PMT with a grease coupling for 0.511 $\mathrm{MeV} \gamma$-rays from a ${ }^{22} \mathrm{Na}$ source with a coincidence trigger. The systematic uncertainty of these measurements is about $1 \%$ [17].

\section{RESULTS AND DISCUSSIONS}

\section{A. Neutron Induced Radiation Damage}

Fig. 2 shows the transmittance spectra measured before (black solid lines) and after (red dashed lines) a $1 \mathrm{MeV}$ equivalent neutron fluence of $1.7,3.4$, and $6.7 \times 10^{15} \mathrm{n}_{\mathrm{eq}} / \mathrm{cm}^{2}$ respectively for the LuAG:Ce samples n-1 (top), n-2 (middle), and n-3 (bottom). Also shown in the figure are the LuAG:Ce emission spectra (blue dashes), the theoretical limit of LuAG:Ce transmittance (blue dots) calculated using the refractive index assuming multiple bounces and no internal absorption [20], and the numerical values of the EWLT for the three samples before (black) and after (red) irradiation. We note that no serious self-absorption in these LuAG:Ce ceramic samples with good optical quality. We also note that the transmittance of these ceramic samples does not approach the theoretical limit because of the scattering centers, such as the pores, inside the sample bulk. While scattering centers do not absorb scintillation light, they may affect the level of radiation induced light output loss because of longer light path. The observed transmittance loss is very small, indicating excellent radiation hardness of the LuAG:Ce samples against neutrons up to $6.7 \times 10^{15} \mathrm{n}_{\mathrm{eq}} / \mathrm{cm}^{2}$.

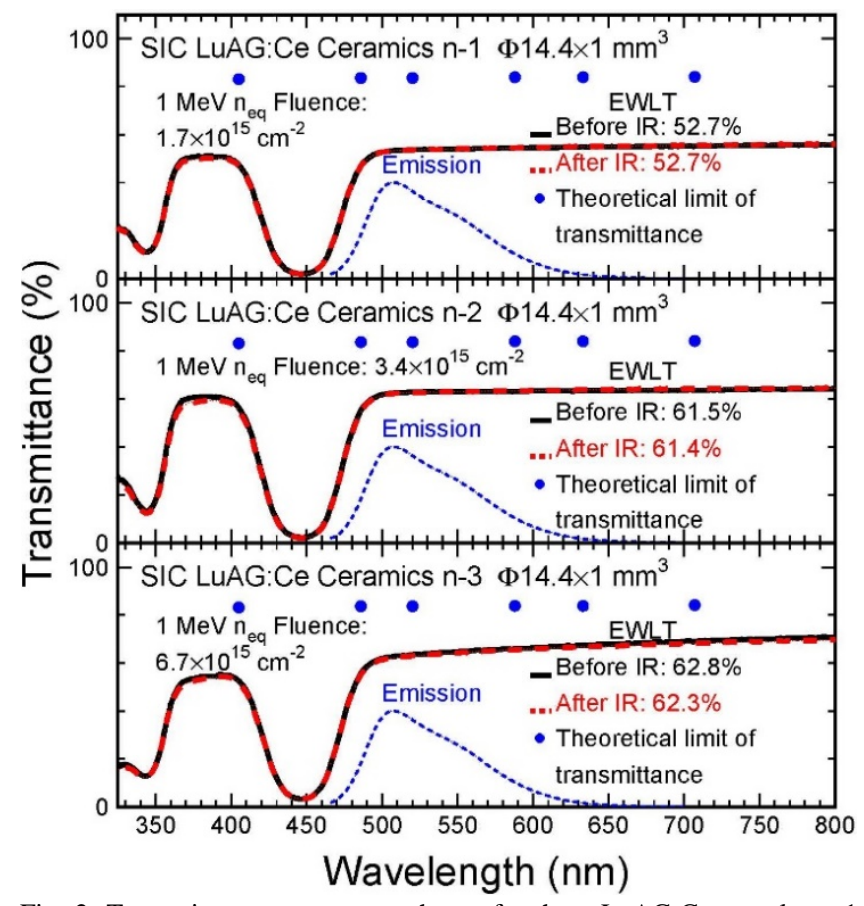

Fig. 2. Transmittance spectra are shown for three LuAG:Ce samples n-1 (top), n-2 (middle), and n-3 (bottom) before (black solid lines) and after (red dash lines) a $1 \mathrm{MeV}$ equivalent neutron fluence of $1.7,3.4$, and $6.7 \times 10^{15}$ $\mathrm{n}_{\mathrm{eq}} / \mathrm{cm}^{2}$ respectively at the East Port in the experiment LANSCE-7638.

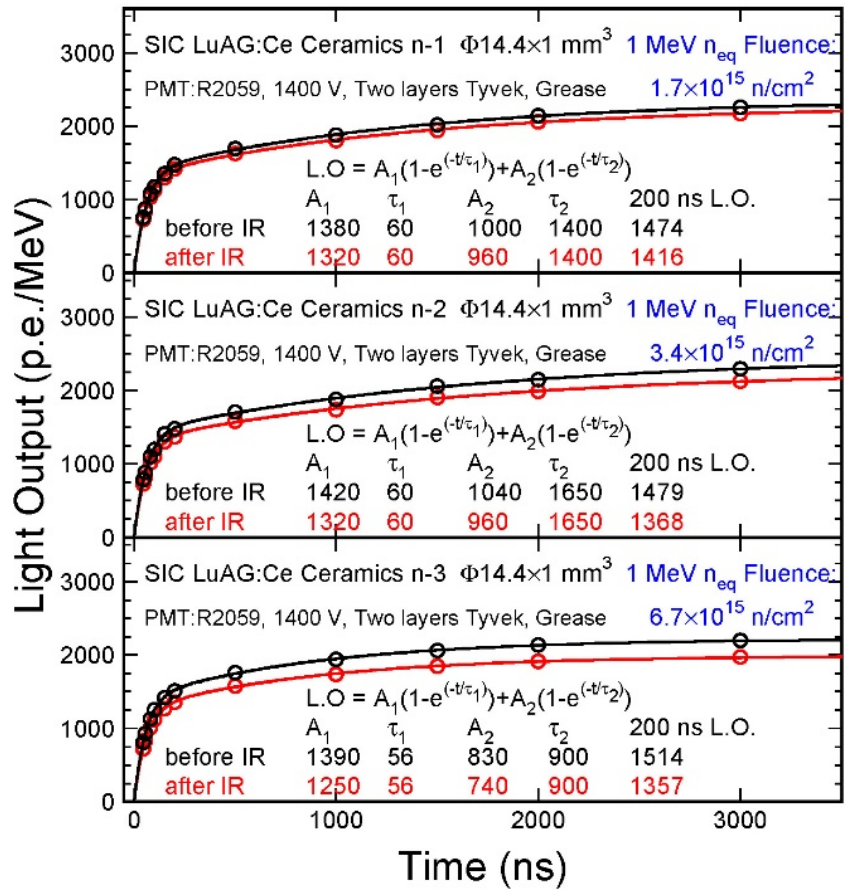

Fig. 3. Light output is shown as a function of the integration time for three LuAG:Ce samples n-1 (top), n-2 (middle), and n-3 (bottom) before (black) and after (red) a $1 \mathrm{MeV}$ equivalent neutron fluence of $1.7,3.4$, and $6.7 \times 10^{15}$ $\mathrm{n}_{\mathrm{eq}} / \mathrm{cm}^{2}$ respectively at the East Port in the experiment LANSCE-7638. 
Fig. 3 shows the light output as a function of the integration time measured before (black) and after (red) a 1 $\mathrm{MeV}$ equivalent neutron fluence of $1.7,3.4$, and $6.7 \times 10^{15}$ $\mathrm{n}_{\mathrm{eq}} / \mathrm{cm}^{2}$ respectively at LANSCE for the LuAG:Ce samples n-1 (top), n-2 (middle), and n-3 (bottom). The observed light output loss is also small, confirming excellent radiation hardness of the LuAG:Ce samples against neutrons. In addition, the $\mathrm{F} / \mathrm{T}$ ratio does not change up to $1 \mathrm{MeV}$ equivalent neutron fluence of $6.7 \times 10^{15} \mathrm{n}_{\mathrm{eq}} / \mathrm{cm}^{2}$.

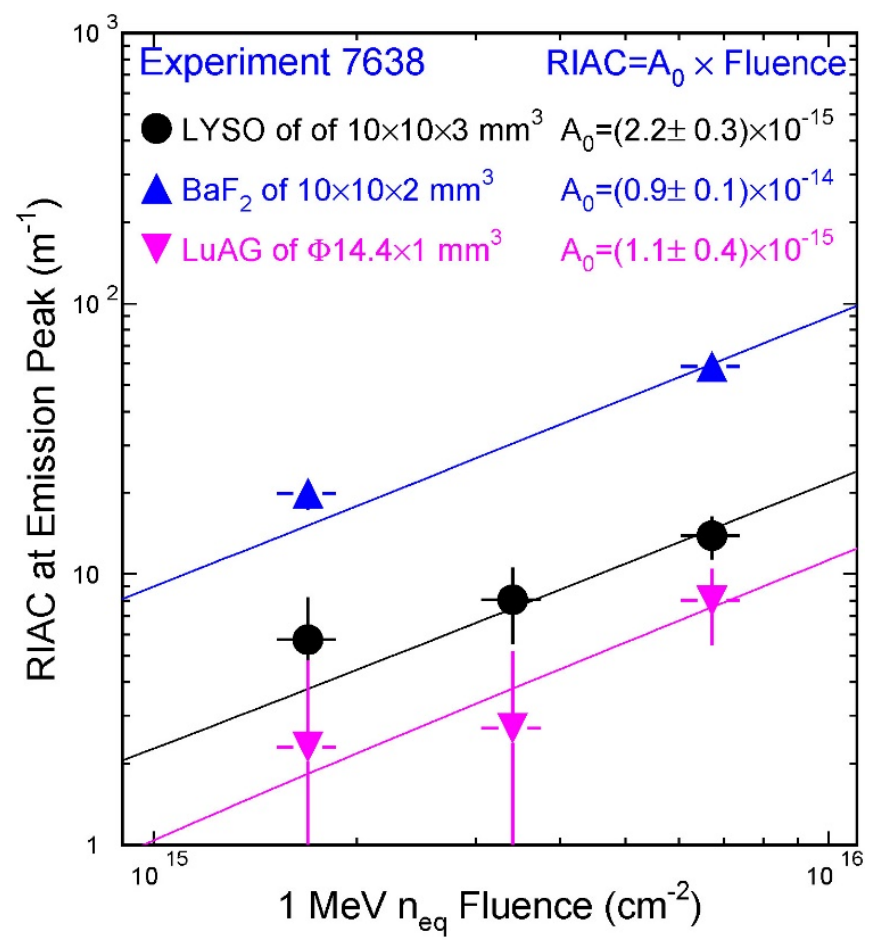

Fig. 4. The RIAC values at the LuAG:Ce emission peak are shown as a function of $1 \mathrm{MeV}$ equivalent neutron fluence for the SIC LYSO (black dots) and $\mathrm{BaF}_{2}$ (blue triangles) crystal samples and three LuAG:Ce (magenta triangles) ceramic samples irradiated at the East Port in the experiment LANSCE-7638.

Fig. 4 shows the RIAC values as a function of the $1 \mathrm{MeV}$ equivalent neutron fluence for LYSO (black dots) and $\mathrm{BaF}_{2}$ (blue triangles) crystals and LuAG:Ce (magenta triangles) ceramic samples irradiated at the East Port in the experiment LANSCE-7638 and the corresponding linear fits. Each RIAC value for $\mathrm{LYSO}$ and $\mathrm{BaF}_{2}$ crystals is an average of two samples irradiated under the same condition, while only one LuAG:Ce sample was irradiated for each fluence. We note that the RIAC value of the LuAG:Ce ceramics against neutrons is about a factor of two smaller than that of LYSO:Ce crystals.

Fig. 5 shows the normalized light output, defined as the light output after irradiation normalized to that before irradiation, as a function of the $1 \mathrm{MeV}$ equivalent neutron fluence for SIC LYSO (black dots) and $\mathrm{BaF}_{2}$ (blue triangles) crystal samples and three LuAG:Ce (magenta triangles) ceramic samples, which were irradiated at the same time at East Port in the experiment LANSCE-7638. We note that all LYSO, $\mathrm{BaF}_{2}$ crystals, and LuAG:Ce ceramics survive a $1 \mathrm{MeV}$ equivalent neutron fluence of $6.7 \times 10^{15} \mathrm{n}_{\mathrm{eq}} / \mathrm{cm}^{2}$ well. The normalized light output values after a $1 \mathrm{MeV}$ equivalent neutron fluence of $6.7 \times 10^{15} \mathrm{n}_{\mathrm{eq}} / \mathrm{cm}^{2}$ is $90 \%$ for the LuAG:Ce ceramic samples, which is higher than LYSO:Ce of $78 \%$ and $\mathrm{BaF}_{2}$ of $81 \%$. The larger light output degradation observed in LYSO and $\mathrm{BaF}_{2}$ crystal samples is partially due to their longer scintillation light path length because of their larger thickness.

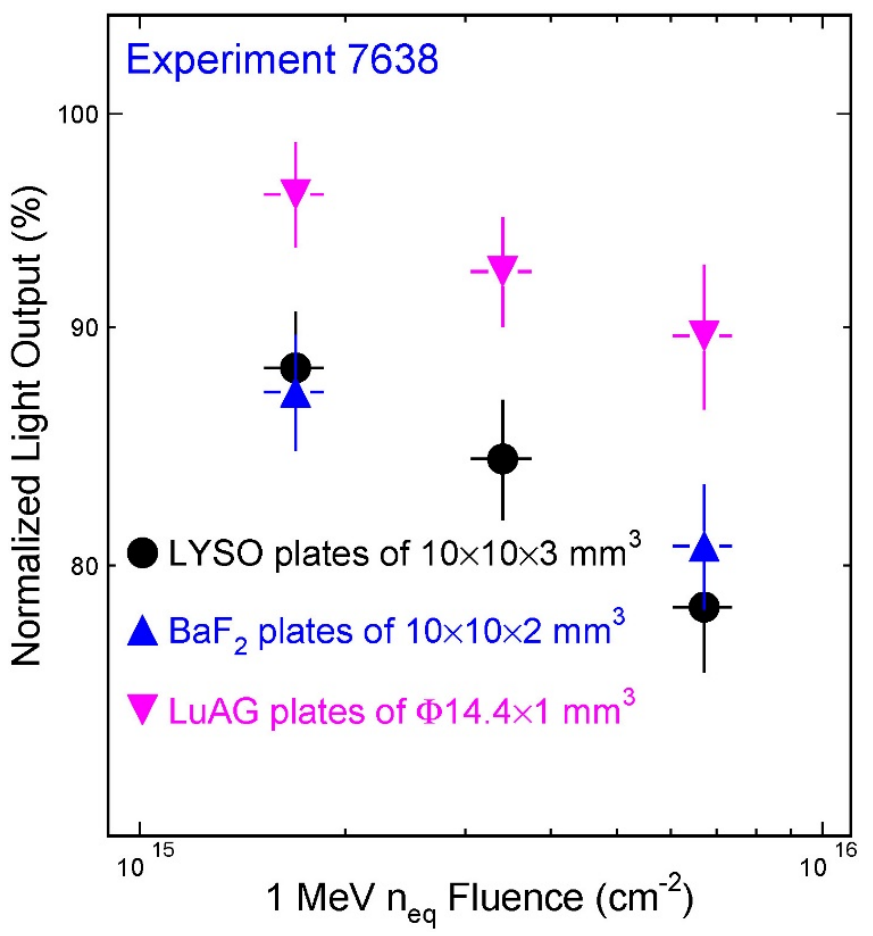

Fig. 5. The normalized light output is shown as a function of $1 \mathrm{MeV}$ equivalent neutron fluence for SIC LYSO (black dots) and $\mathrm{BaF}_{2}$ (blue triangles) crystal samples and LuAG:Ce (magenta triangles) ceramic samples irradiated at the East Port in the experiment LANSCE-7638.

\section{B. Proton Induced Radiation Damage}

Fig. 6 shows the transmittance spectra measured before (black solid lines) and after (red dashed lines) a proton fluence of $1.2 \times 10^{15} \mathrm{p} / \mathrm{cm}^{2}$ at CERN PS-IRRAD (top) and $2.3 \times 10^{14}$ $\mathrm{p} / \mathrm{cm}^{2}$ at LANSCE (bottom), respectively, for the $\mathrm{Mg}^{2+}$ codoped LuAG:Ce ceramic sample SIC p-3 (top), and the $\mathrm{Ca}^{2+}$ and $\mathrm{Mg}^{2+}$ co-doped LuAG:Ce ceramic sample SIOM p-5 (bottom). Also shown in the figure are the LuAG:Ce emission spectra (blue dashes), the theoretical limit of transmittance (blue dots), and the numerical values of the EWLT before (black) and after (red) irradiation.

We note their poor optical quality shown as low transmittance caused by scattering centers in these LuAG:Ce ceramic samples, which affect more transmittance at shorter wavelengths.

Once again, the observed degradation of transmittance is very small, confirming excellent radiation hardness of the LuAG:Ce ceramic scintillators against protons up to $1.2 \times 10^{15}$ $\mathrm{p} / \mathrm{cm}^{2}$. 


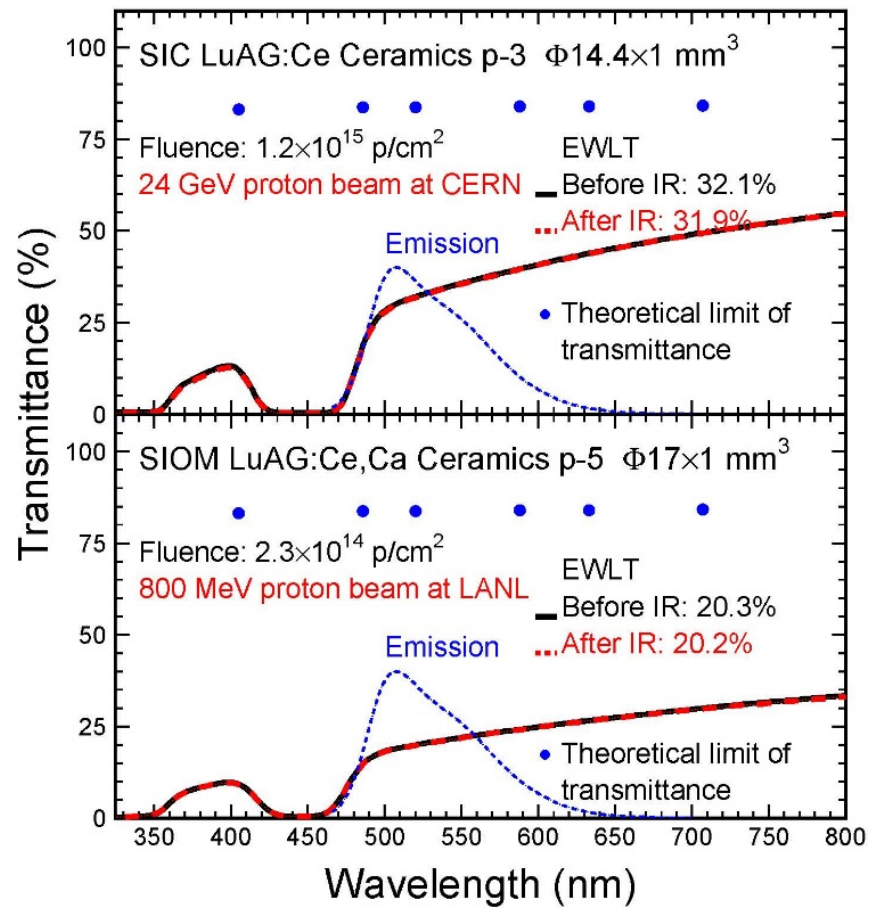

Fig. 6. The transmittance spectra are shown for the $\mathrm{Mg}^{2+}$ co-doped LuAG:Ce ceramic sample SIC p-3 (top), and the $\mathrm{Mg}^{2+}$ and $\mathrm{Ca}^{2+}$ co-doped LuAG:Ce ceramic sample SIOM p-5 (bottom) before (black) and after (red) a proton fluence of $1.2 \times 10^{15} \mathrm{p} / \mathrm{cm}^{2}$ at CERN PS-IRRAD (top) and $2.3 \times 10^{14} \mathrm{p} / \mathrm{cm}^{2}$ at LANSCE (bottom), respectively.

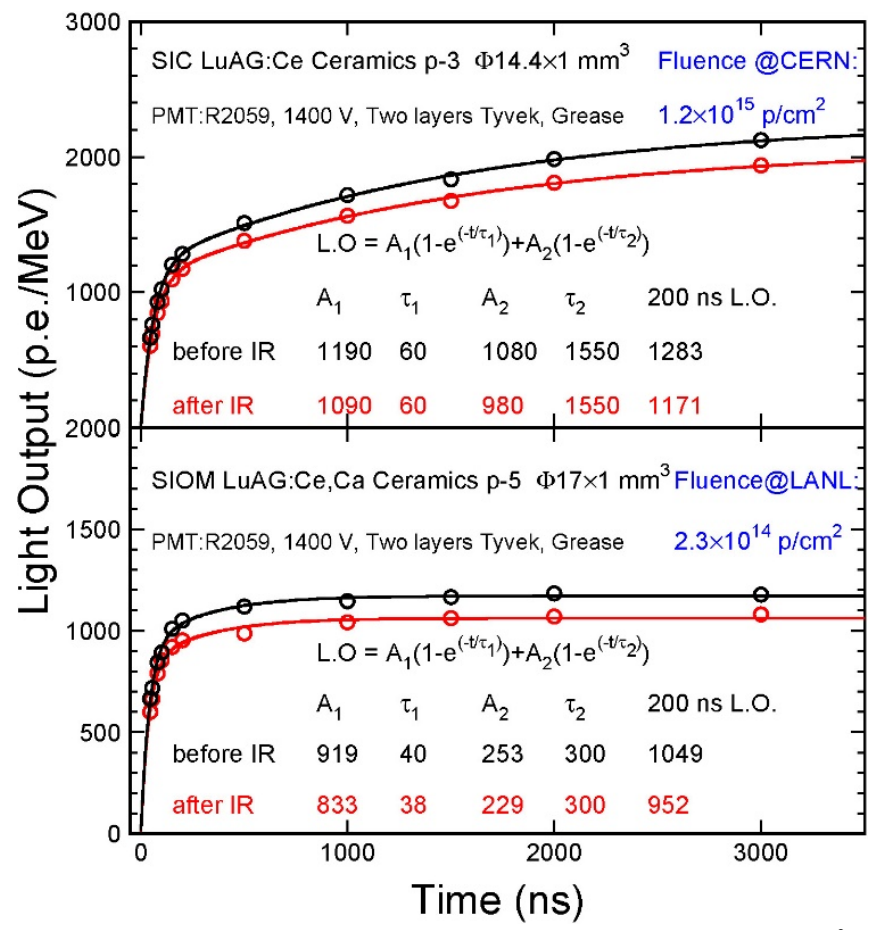

Fig. 7. Light output is shown as a function of integration time for the $\mathrm{Mg}^{2+}$ co-doped LuAG:Ce ceramic sample SIC p-3 (top), and the $\mathrm{Mg}^{2+}$ and $\mathrm{Ca}^{2+}$ co-doped LuAG:Ce ceramic sample SIOM p-5 (bottom) before (black) and after (red) a proton fluence of $1.2 \times 10^{15} \mathrm{p} / \mathrm{cm}^{2}$ at CERN PS-IRRAD (top) and $2.3 \times 10^{14} \mathrm{p} / \mathrm{cm}^{2}$ at LANSCE (bottom), respectively.

Fig. 7 shows the light output as a function of integration time for the $\mathrm{Mg}^{2+}$ co-doped SIC LuAG:Ce ceramic sample p-3 (top), and the $\mathrm{Mg}^{2+}$ and $\mathrm{Ca}^{2+}$ co-doped SIOM LuAG:Ce ceramic sample p-5 (bottom) before (black) and after (red) a proton fluence of $1.2 \times 10^{15} \mathrm{p} / \mathrm{cm}^{2}$ at CERN PS-IRRAD Proton Facility (top) and $2.3 \times 10^{14} \mathrm{p} / \mathrm{cm}^{2}$ at the blue room of LANSCE (bottom), respectively. Once again, about $90 \%$ light remains for both samples. We note that the $\mathrm{F} / \mathrm{T}$ ratio does not change for both samples. We also note that the $\mathrm{Mg}^{2+}$ co-doping improves light output, and the $\mathrm{Ca}^{2+}$ and $\mathrm{Mg}^{2+}$ co-doping improves the $\mathrm{F} / \mathrm{T}$ ratio.

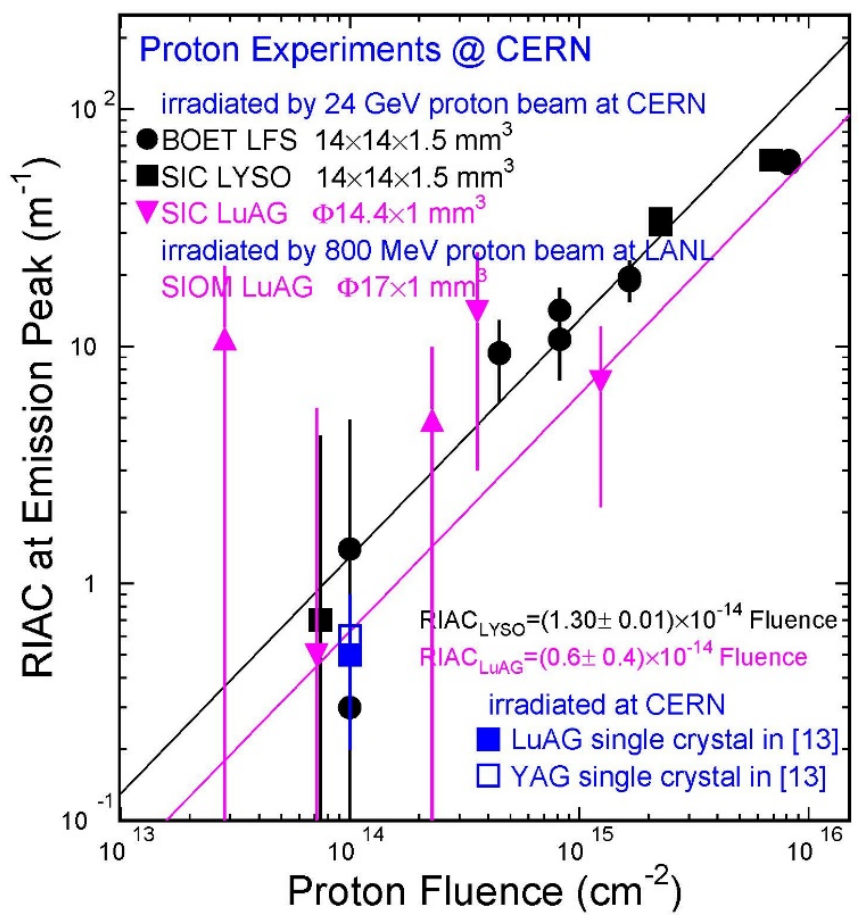

Fig. 8. The RIAC values as a function of the proton fluence for the LYSO/LFS (black dots) crystal and LuAG:Ce (magenta triangles) ceramic samples irradiated by both $24 \mathrm{GeV}$ protons at CERN PS-IRRAD and 800 $\mathrm{MeV}$ protons at LANL and the corresponding linear fits.

Fig. 8 shows the RIAC values as a function of the proton fluence for the LYSO/LFS (black dots) crystal and LuAG:Ce (magenta triangles) ceramic samples irradiated by $24 \mathrm{GeV}$ and $800 \mathrm{GeV}$ protons at CERN PS-IRRAD and the Blue Room of LASCE, respectively, and the corresponding linear fits. The results for the SIC LYSO and BOET (Beijing Opto-Electronics Technology Co.) LFS crystals were reported in our previous publications [21, 22]. Only one sample was irradiated for each data point.

We note that the proton induced RIAC values for the LuAG:Ce ceramic samples with good initial transmittance, e.g., samples p-1 and p-3, appear also about a factor of two smaller than that of LYSO crystals. We also note that proton induced RIAC values are large and with large error bars for some LuAG:Ce ceramic samples, e.g., p-2, p-4, and p-5, which is due to their poor optical quality. Also shown in the figure is the fit result of RIAC $\left(\mathrm{m}^{-1}\right)=(0.6 \pm 0.4) \times 10^{-14} \mathrm{~F}_{\mathrm{p}}$ which agrees well with $0.5 \pm 0.3 \mathrm{~m}^{-1}$ reported in the reference [13] for LuAG crystal samples after $10^{14} \mathrm{~F}_{\mathrm{p}}$ by $24 \mathrm{GeV}$ protons. This factor of two is consistent with the neutron irradiation data shown in Fig. 4.

Fig. 9 shows normalized light output as a function of the proton fluence for the LYSO/LFS crystals (black dots) and 
LuAG:Ce ceramic samples (magenta triangles) irradiated by 24 $\mathrm{GeV}$ and $800 \mathrm{MeV}$ protons at CERN PS-IRRAD and the Blue Room of LANSCE respectively. Both LYSO crystals and LuAG:Ce ceramics survive a proton fluence of $10^{15} \mathrm{p} / \mathrm{cm}^{2}$. The normalized light output value after a proton fluence of $1.2 \times 10^{15}$ $\mathrm{p} / \mathrm{cm}^{2}$ is $91 \%$ for the LuAG:Ce ceramic samples, which is about the same values as the $90 \%$ observed for SIC LYSO crystals after a proton fluence of $9.7 \times 10^{14} \mathrm{p} / \mathrm{cm}^{2}$. LuAG:Ce ceramic thus may be considered as another promising inorganic scintillator for future HEP experiments in a severe radiation environment, such as the HL-LHC and the proposed FCC-hh.

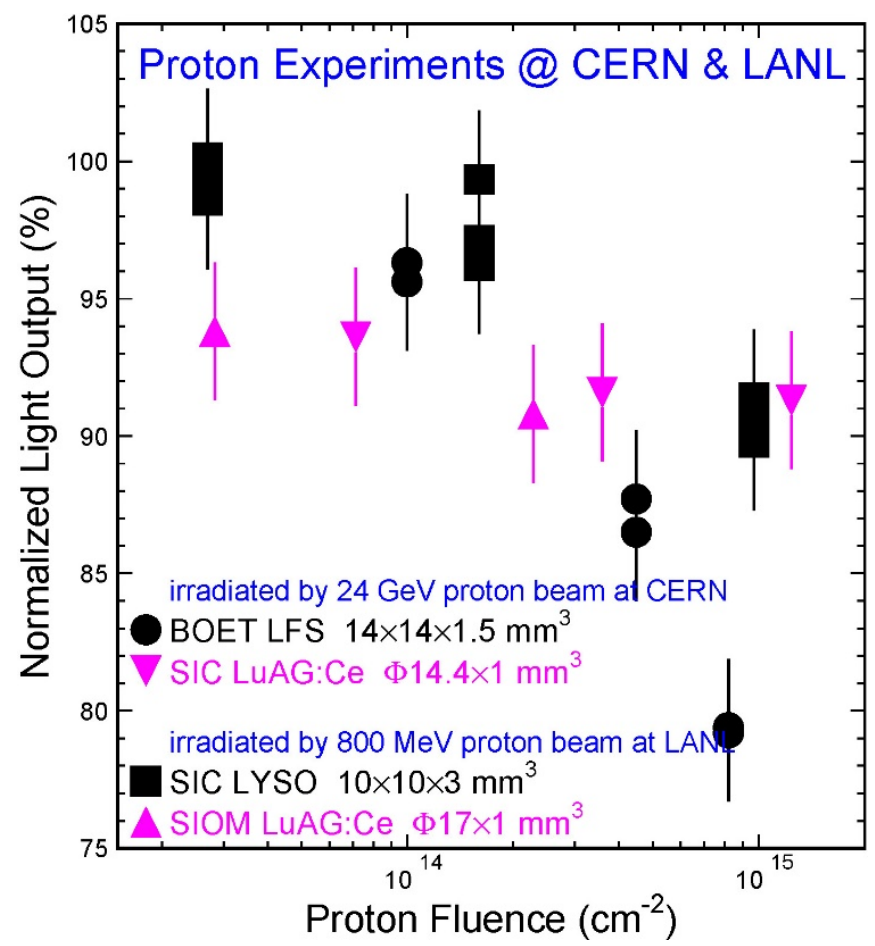

Fig. 9. Normalized light output is shown as a function of proton fluence for the LYSO/LFS crystals (black dots) and LuAG:Ce (magenta triangles) ceramic samples irradiated by $24 \mathrm{GeV}$ protons at CERN PS-IRRAD and by $800 \mathrm{MeV}$ protons at LANSCE.

\section{SUMMARY}

$\mathrm{Ca}^{2+}$ and $\mathrm{Mg}^{2+}$ co-doped LuAG:Ce ceramic samples were fabricated and irradiated by neutrons at the East Port of LANSCE up to $6.7 \times 10^{15} \mathrm{n}_{\mathrm{eq}} / \mathrm{cm}^{2}$ and by $24 \mathrm{GeV}$ and $800 \mathrm{MeV}$ protons at the CERN PS-IRRAD up to $1.2 \times 10^{15} \mathrm{p} / \mathrm{cm}^{2}$ and at the Blue Room of LANSCE up to $2.3 \times 10^{14} \mathrm{p} / \mathrm{cm}^{2}$, respectively. $\mathrm{Mg}^{2+}$ co-doping in LuAG:Ce ceramics improves light output, while $\mathrm{Ca}^{2+}$ and $\mathrm{Mg}^{2+}$ co-doping improves the $\mathrm{F} / \mathrm{T}$ ratio.

The RIAC values of LuAG:Ce ceramics were found to have a factor of two smaller than LYSO:Ce crystals against neutrons. Similar result was also obtained against protons for the LuAG:Ce ceramic samples with good optical quality. We plan to irradiate LuAG:Ce ceramic samples of improved optical quality to reduce the uncertainties in the RIAC data in our future investigation.
More than $90 \%$ of the light output remains in $1 \mathrm{~mm}$ thick samples after fluences of up to $6.7 \times 10^{15} \mathrm{n}_{\mathrm{eq}} / \mathrm{cm}^{2}$ and $1.2 \times 10^{15}$ $\mathrm{p} / \mathrm{cm}^{2}$ LuAG:Ce ceramic scintillators which makes this material promising for applications at the HL-LHC and the proposed FCC-hh.

$\mathrm{R} \& \mathrm{D}$ will continue to develop $\mathrm{Ca}^{2+}$ and $\mathrm{Mg}^{2+}$ co-doped LuAG:Ce ceramic scintillators with improved optical quality, $\mathrm{F} / \mathrm{T}$ ratio and radiation hardness.

\section{ACKNOWLEDGEMENTS}

The authors are grateful to LANSCE and the CERN PSIRRAD proton facility for providing $800 \mathrm{MeV}$ and $24 \mathrm{GeV}$ proton beams respectively used in this investigation.

\section{REFERENCES}

[1] H.-L. Li, X.-J. Liu, R.-J. Xie, et al., "Fabrication of transparent ceriumdoped lutetium aluminum garnet ceramics by co-precipitation routes," $J$. Am. Ceram. Soc., vol. 89, no. 7, pp. 2356-2358, May 2006.

[2] N. J. Cherepy, J. D. Kuntz, T. M. Tillotson, et al., "Cerium-doped single crystal and transparent ceramic lutetium aluminum garnet scintillators," Nucl. Instrum. Methods Phys. Res. A vol. 579, no. 1, pp. 38-41, Aug. 2007.

[3] Y. Wang, G. Baldoni, W. H. Rhodes, et al., "Transparent Garnet Ceramic Scintillators for Gamma-ray Detection,” in Proc. SPIE 8507, Hard X-Ray, Gamma-Ray, and Neutron Detector Physics XIV, pp. 850717, 2012.

[4] T. Yanagida, Y. Fujimoto, Y. Yokota, et al., "Scintillation properties of LuAG (Ce) ceramic and single crystalline scintillator," in IEEE Nuclear Science Symposium \& Medical Imaging Conference, pp. 1612-1614, 2010.

[5] T. Yanagida, Y. Fujimoto, K. Kamada, et al., "Scintillation Properties of Transparent Ceramic Pr:LuAG for Different Pr Concentration,” IEEE Trans. Nucl. Sci., vol. 59, no. 5, pp. 2146 - 2151, Oct. 2012.

[6] C. Hu, J. Li, F. Yang, et al., "LuAG ceramic scintillators for future HEP experiments," Nucl. Instrum. Methods Phys. Res. A vol. 954, pp. 161723, Feb. 2020.

[7] S. Liu, X. Feng, Z. Zhou, et al., "Effect of $\mathrm{Mg}^{2+}$ co-doping on the scintillation performance of LuAG:Ce ceramics," Phys. Status Solidi Rapid Res. Lett., vol. 8, no. 1, pp. 105-109, Jan. 2014.

[8] M. Nikl, K. Kamada, V. Babin, et al., "Defect engineering in Ce-doped aluminum garnet single crystal scintillators," Cryst. Growth Des., vol. 14, no. 9, pp. 4827-4833, Jul. 2014.

[9] A.G. Petrosyan, K.L. Ovanesyan, M.V. Derdzyan, et al., "A study of radiation effects on LuAG:Ce(Pr) co-activated with Ca," J. Cryst. Growth, vol. 430, pp. 46-51, Nov. 2015.

[10] B. Bilki and The CMS Collaboration, "CMS forward calorimeters phase II upgrade,” J. Phys., Conf. Ser., vol. 587, Feb. 2015, Art. no. 012014.

[11] M.V. Derdzyan, K.L. Ovanesyan, A.G. Petrosyan, et al., "Radiation hardness of LuAG:Ce and LuAG:Pr scintillator crystals," J. Cryst. Growth, vol. 361, pp. 212-216, Dec. 2012.

[12] Y. Shen, X. Feng, Y. Shi, et al., "The radiation hardness of Pr:LuAG scintillating ceramics,” Ceram. Int., vol. 40, no. 2, pp. 3715-3719, Mar. 2014.

[13] M. T. Lucchini, K. Pauwels, K. Blazek, et al., "Radiation tolerance of LuAG:Ce and YAG:Ce crystals under high levels of gamma- and protonirradiation," IEEE Trans. Nucl. Sci., vol. 63, no. 2, pp. 586 - 590, Apr. 2016.

[14] X. Chen, Z. Hu, M. Cao, et al., "Influence of cerium doping concentration on the optical properties of Ce,Mg:LuAG scintillation ceramics," J. Eur. Ceram. Soc., vol. 38, no. 9, pp. 3246-3254, Aug. 2018.

[15] W. Ma, B. Jiang, and S. Chen, et al., "A fast lutetium aluminum garnet scintillation ceramic with $\mathrm{Ce}^{3+}$ and $\mathrm{Ca}^{2+}$ co-dopants," J. Lumin., vol. 216, pp. 116728, Dec. 2019.

[16] R. Boulesteix, A. Maître, J.-F. Baumard, Y. Rabinovitch, and F. Reynaud, "Light scattering by pores in transparent Nd:YAG ceramics for lasers: correlations between microstructure and optical properties," Opt. Express, vol. 18, no. 14, pp. 14992-15002, June 2010.

[17] C. Hu, F. Yang, L. Zhang, et al., "Neutron-Induced Radiation Damage in LYSO, $\mathrm{BaF}_{2}$, and PWO Crystals,” IEEE Trans. Nucl. Sci., vol. 67, no. 6, pp. 1086-1092, June 2020. 
[18] LANL and CERN for Science Community. [Online]. Available: https://laws.lanl.gov/vhosts/mcnp.lanl.gov/index.shtm

[19] LANL and CERN for Science Community. [Online]. Available: https://rd50.web.cern.ch/rd50/NIEL/default.html

[20] D.-A. Ma and R.-Y. Zhu, "Light attenuation length of barium fluoride crystals," Nucl. Instrum. Methods Phys. Res. A, Accel. Spectrum. Detect. Assoc. Equip., vol. 333, nos. 2-3, pp. 422-424, Sep. 1993.

[21] F. Yang, L. Zhang, R.-Y. Zhu, et al., "Proton-Induced Radiation Damage in Fast Crystal Scintillators," IEEE Trans. Nucl. Sci., vol. 64, no. 1, pp. 665-672, Jan. 2017.

[22] C. Hu, F. Yang, L. Zhang, et al., "Proton-Induced Radiation Damage in $\mathrm{BaF}_{2}$, LYSO, and PWO Crystal Scintillators,” IEEE Trans. Nucl. Sci., vol. 65, no. 4, pp. 1018 - 1024, Apr. 2018. 